\title{
Effectiveness of Selected Procedures of Hearing Aid Adaptation - Clinical Cases
}

\author{
D. HOJAN-JEZIERSKA ${ }^{a}$ AND A. OLEJNIK ${ }^{b, *}$ \\ ${ }^{a}$ Dept. of Biophysics, Poznań Univ. of Medical Sciences, Fredry 10, 61-701 Poznań, Poland \\ ${ }^{b}$ Institute of Acoustics, A. Mickiewicz Univ., Umultowska 85, 61-614 Poznań, Poland
}

For five patients representing different clinical cases the effectiveness of hearing aids adjusted to the individual hearing impairment and with the electroacoustic parameters established on the basis of different algorithms has been evaluated. The results permitted formulation of the rules optimising the effectiveness of the hearing aid use, especially in speech intelligibility.

PACS numbers: 43.66.Ts

\section{Introduction}

The hearing aid should amplify the acoustic signals to make them perceptible (by adjustment of the loudness) and should ensure the optimum sound quality so that the speech signal would be understood in different acoustic conditions [1-8]. To fulfil its function the electroacoustic characteristics of the hearing aid must be correctly adjusted on the basis of specific algorithms taking into account the type of hearing impairment of the patient [9-16]. The majority of the so far proposed algorithms have been based on the results of the classical tonal audiometry, usually not analysed exactly in relation to the type of hearing impairment of the patient. Another problem was the systematisation of the adjustment rules to ensure the optimisation of the hearing aid functioning, especially in the speech intelligibility. This study was undertaken as an attempt at formulation of specific rules for the optimum adjustment of hearing aids.

\section{Subject, hearing instruments and the method}

The type and scale of hearing impairment were determined on the basis of the patient's examination in:

- tonal audiometry

- speech audiometry

- The methods of hearing aid adjustment were divided into two groups according to the scale of the hearing loss [1].

- Small and medium hearing loss - Berger rule, NAL-RP rule (National Acoustical Laboratory Revision Procedure), $\mathrm{DSL}_{\mathrm{i} / \mathrm{o}}$ rule

- Large hearing loss - POGO 2 rule, NAL-NL1 rule (National Acoustical Laboratory - Non Linear 1), $\mathrm{DSL}_{\mathrm{i} / \mathrm{o}}$ rule

\footnotetext{
* corresponding author; e-mail: agamon63@wp.pl
}

- Hearing aids were selected and adjusted to individual patients according to certain rules and taking into regard the type and scale of hearing impairment. After adjustment of the hearing aid according to a given rule the patient was asked to use it for one week in the normal everyday conditions. In next week the effectiveness of the hearing aid adjustment was evaluated.

- Two procedures were applied for evaluation of the effectiveness of the hearing aid adjustment APHAB (Abbreviated Profile of Hearing Aid Performance) [10].

- The procedure of speech intelligibility evaluation in free field [4].

- Hearing aids used in the study:

Hearing aid 1

— digital

- amplification control in 2 channels

$-\mathrm{AGC}_{i}$

- anti-feedback manager

— transmission band 200-5200 Hz

- 3 programs of hearing

Hearing aid 2

- digital

- amplification control in 6 channels

- $\mathrm{AGC}_{i}$

- anti-feedback manager

- transmission band 200-6000 Hz

- 3 programs of hearing

- noise reduction 


\section{Results and discussion}

For five patients representing different clinical cases the effectiveness of hearing aids adjusted to the individual hearing impairment and with the electroacoustics parameters established on the basis of different algorithms has been evaluated.

\section{- Patient 1}

Age 25, sensorneural hearing loss in the right ear $(\mathrm{HTL}=52 \mathrm{~dB})$, progressing from the 8th year of age when appeared as a result of the scull injury; in the left year residual hearing loss $(\mathrm{HTL}=90 \mathrm{~dB})$. The patient did not use any hearing aid prior to the study, Fig. 1.

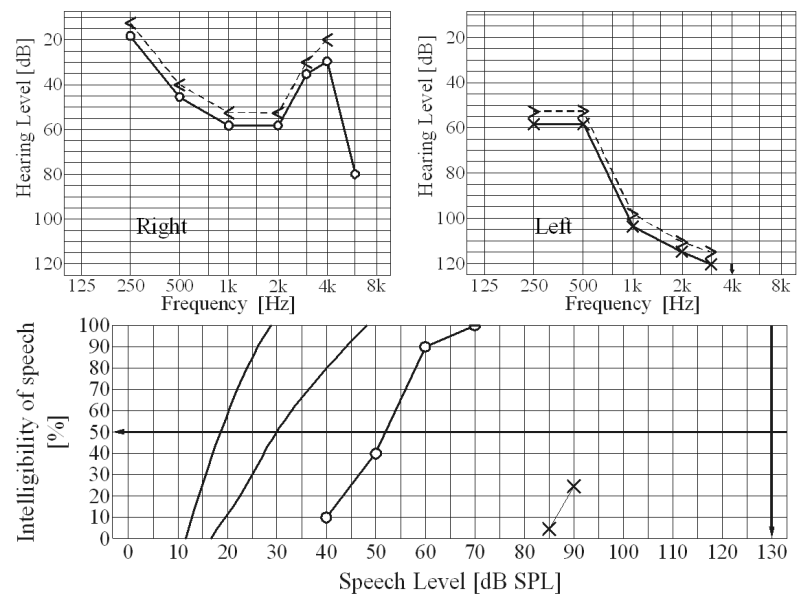

Fig. 1. Audiometric data for patient 1.

During the study the patient 1 was wearing a hearing aid in the right ear only (the patient's own hearing aid is always labelled as (3). Hearing aid 1 for the right ear was adjusted according to the Berger rule and later according to the NAL-RP rule. Hearing aid 3 for the right ear was adjusted according to the NAL-NL1 rule.

Evaluation of the effectiveness of the hearing aids for patient 1 is illustrated in Figs. 2-3.

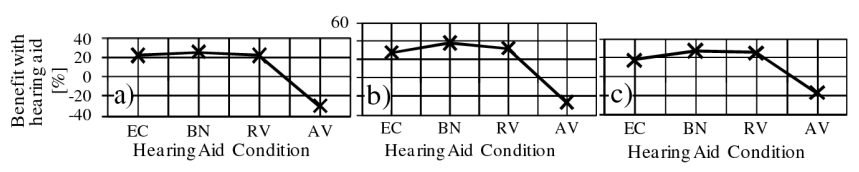

Fig. 2. Self-reported hearing aid outcome for hearing aid 1 [Berger rule (a), NAL-RP rule (b)] and hearing aid 3 [NAL-NL1 rule (c)], measured using the four subscales of the APHAB procedure. Ease of communication (EC), background noise $(\mathrm{BN})$, reverberation (RV), aversiveness (AV).

Subjective evaluation by the patient; comments

- Adjustment according to the Berger rule:

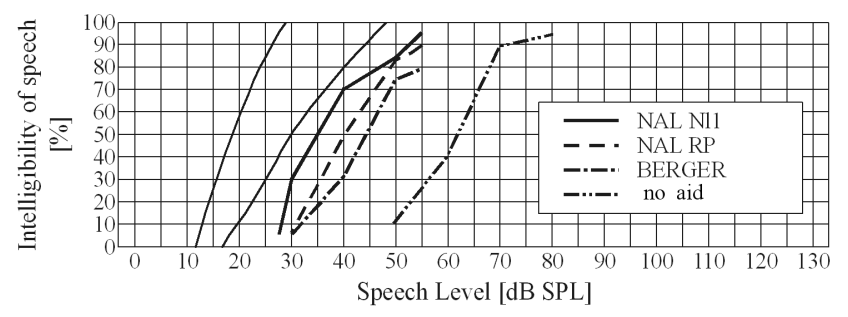

Fig. 3. The speech intelligibility evaluation.

- Poorer speech intelligibility in background noise

- Unpleasant reception of loud sounds

- Problems with perception of silent sounds

- Adjustment according to NAL-RP rule:

- Improved speech intelligibility

- Less unpleasant perception of loud sounds

- Comments:

According to the patient opinion expressed in the APHAB protocol (Fig. 2), he had the highest comfort of hearing evaluated on the basis of the attributes EC, BN, and AV with the hearing aid adjusted according to the NAL-RP rule, the comfort was poorer with the hearing aid adjusted according to NAL-NL1 rule.

Taking into regard the speech intelligibility criterion (Fig. 3), the best results were obtained for the hearing aid adjusted according to NAL-NL1 rule.

The lower comfort of hearing, evaluated on the basis of these attributes, was obtained with the hearing aid adjusted according to the Berger rule.

\section{- Patient 2}

Age 24, sensorneural hearing loss $\mathrm{HTL}=75 \mathrm{~dB}$ in the left ear and $\mathrm{HTL}=85 \mathrm{~dB}$ in the right ear, from the birth treated by ototoxic medicaments, cerebral palsy, from the 6 th year of age has been wearing a hearing aid in the left ear only.

\section{- Comments:}

As follows from analysis of APHAB questionnaire, the highest comfort of hearing the patient had with the hearing aid adjusted according to the POGO 2 rule, known to give good results when applied to linear hearing aids. A bit lower was the hearing comfort achieved with the hearing aid adjusted according to the NAL-NL1 rule. The lowest evaluation of the hearing aid adjusted according to $\mathrm{DSL}_{\mathrm{i} / \mathrm{o}}$ followed from the unpleasant perception of loud sounds (AV attribute).

The best speech intelligibility was achieved with the hearing aids adjusted according to the NAL-NL1 rule, while the results obtained when applying the rules POGO 2 and $\mathrm{DSL}_{\mathrm{i} / \mathrm{o}}$ were similar but indicated poorer speech intelligibility. 
- Patient 3

Age 75, hearing loss of the presbyacusis type related to the earlier profession (acoustic injury); light hearing loss $(\mathrm{HTL}=32 \mathrm{~dB}$ in the left ear and HTL $=22 \mathrm{~dB}$ in the right ear).

- Comments:

As follows from the patients subjective evaluation expressed in APHAB terms and in speech intelligibility tests, no significant differences in the sound perception were indicated for the hearing aid adjusted according to different rules. A significantly poorer performance was noted for the hearing aid adjusted according to POGO 1 applied in the patient's own hearing aid, however, the result may be unreliable taking into regard the hearing aid quality.

\section{- Patient 4}

Age 59, sensorineural hearing loss, medium size with recruitment, $(\mathrm{HTL}=45 \mathrm{~dB}$ in the left ear and HTL $=$ $48 \mathrm{~dB}$ in the right ear) as a result of acoustic injury, a hearing aid for 9 years has been using.

- Comments:

In the evaluation of the patient expressed in APHAB questionnaire, the best performance had the hearing aid adjusted according to $\mathrm{DSL}_{\mathrm{i} / \mathrm{o}}$, which offered much higher comfort of hearing than the adjustment according to the Berger and POGO 1 rules. The same highest evaluation got the hearing aid adjusted according to $\mathrm{DSL}_{\mathrm{i} / \mathrm{o}}$ rule as far as the speech intelligibility was concerned.

\section{- Patient 5}

Age 60, medium size sensor neural hearing loss HTL $=$ $60 \mathrm{~dB}$ in the left ear and HTL $=53 \mathrm{~dB}$ in the right ear) hearing impairment of genetic origin from early childhood.

\section{- Comments:}

As follows from the patient's evaluation expressed in $\mathrm{APHAB}$ questionnaire, the best performance was achieved with the hearing aid adjusted according to the Berger rule. The high evaluation could result from the difference in the sound quality offered by the patient's own hearing aid and the hearing aid offered to use during the study.

According to the criteria based on the three attributes $\mathrm{EC}, \mathrm{BN}$, and RV, the hearing aids adjusted according to NAL-NL1, DSL $\mathrm{i}_{\mathrm{i} / \mathrm{o}}$, and also POGO 2 (in the patient's own hearing aid) rules offered similar quality of performance. However, only the hearing aid adjusted according to $\mathrm{DSL}_{\mathrm{i} / \mathrm{o}}$ rule offered pleasant perception of loud and unexpected sounds.

According to the criteria of speech intelligibility the hearing aids adjusted according to the Berger and $\mathrm{DSL}_{\mathrm{i} / \mathrm{o}}$ rules, offered similar performance better than those adjusted according to NAL-NL1 and POGO 2 rules. The patient's evaluation coincides with the results expressed in APHAB questionnaire and speech intelligibility, giving in general the highest appreciation to the hearing aid adjusted according to $\mathrm{DSL}_{\mathrm{i} / \mathrm{o}}$ rule.

\section{Final conclusions}

Subjective evaluation of the hearing aid performance by the patients with sensorneural hearing loss, without recruitment, indicated the highest hearing comfort for the hearing aid adjusted according to the rules NAL-NL1 and NAL-RP. The hearing aid adjustment according to the Berger and POGO 2 rules was described as giving much poorer hearing comfort.

The adjustment according to the $\mathrm{DSL}_{\mathrm{i} / \mathrm{o}}$ rule has brought the best results to the patients with sensorneural hearing loss and recruitment as well as to those suffering hearing loss as a consequence of acoustic injury.

The hearing aid adjustment according to the Berger rule has offered high comfort of hearing to patients with small hearing loss of the presbyacusis type.

\section{References}

[1] D. Byrne, Monographs in Contemporary Audiol 4(1), (1983).

[2] H. Dillon, Hörakustik 9, (2000).

[3] D. Hayes, D.A. Eddins, M. Dörscher, Hörakustik 1, 14 (2009).

[4] S. Kochkin, Hearing Journal, 58, 9 (2005).

[5] R. Kwiatkowski, E. Hojan, W. Szyfter, A. Furmann, D. Hojan-Jezierska, Archives of Acoustic 33, 423 (2008).

[6] J.H. Macrae, H. Villon, J. of Rehab Res Dev. 33, 363 (1996).

[7] T. Pötzl, Hörakustik 4, (2007).

[8] M. Schaarschmidt, Hörakustik 8, 84 (2007).

[9] D. Hojan-Jezierska, E. Hojan, Physica Medica; XX, Supl. 1, 135 (2004).

[10] E. Hojan, H. Fastl, J. Malenda, D. Hojan-Jezierska, Archives of Acoustics 30, 1 (2005).

[11] E. Hojan, A. Furmann, L. Chmielewska, D. Hojan-Jezierska, Archives of Acoustics 30 (Supplement), 245 (2005).

[12] D. Hojan-Jezierska, O.M. Stieler, Polish J. of Environmental Studies 15, 53 (2006).

[13] D. Hojan-Jezierska, E. Skrodzka, E. Hojan, Polish J. of Environmental Studies 15, 56 (2006).

[14] D. Hojan-Jezierska, E. Skrodzka, E. Hojan, Archives of Acoustics Vol. 32, 3, 441 (2007).

[15] D. Hojan-Jezierska, E. Skrodzka, Polish J. of Medical Physics and Engineering (2009) (in press).

[16] J. Kiessling, M. Schubert, Scand. Audiol. 25, 153 (1996).

[17] W. Geers, E. Hojan, D. Hojan-Jezierska, Applied Acoustics 51, 199 (1997). 